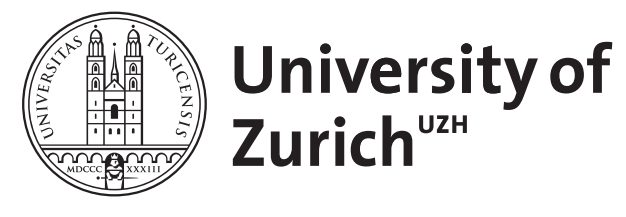

\title{
Kaudalanästhesie bei Kindern
}

\author{
Mauch, J ; Weiss, M
}

\begin{abstract}
Caudal block is a safe procedure commonly used for pediatric perioperative analgesia. Complications are extremely rare but nevertheless local and systemic contraindications must be excluded. Optimal safety and quality result when strict attention is paid to technical details. A local anesthetic (LA) containing epinephrine allows early detection of inadvertent intravascular LA administration; therefore an epinephrine/LA mixture is recommended at least for the test dose. In terms of safety the choice of LA itself is probably of secondary importance. Clonidine as an adjuvant has an excellent risk/benefit profile with minimal side effects. Inadvertent systemic LA intoxication is a rare but potentially fatal complication of regional anesthesia and measures for prevention and early detection are essential. Should circulatory arrest occur, immediate resuscitation following standard guidelines is to be initiated including the use of epinephrine as the first line drug. Intravenous administration of lipid solutions may be beneficial as a secondary adjunct to stabilize hemodynamics but is not an alternative to epinephrine.
\end{abstract}

DOI: https://doi.org/10.1007/s00482-012-1202-0

Other titles: Pediatric caudal anesthesia: importance and aspects of safety concerns

Posted at the Zurich Open Repository and Archive, University of Zurich

ZORA URL: https://doi.org/10.5167/uzh-67327

Journal Article

Published Version

Originally published at:

Mauch, J; Weiss, M (2012). Kaudalanästhesie bei Kindern. Der Schmerz, 26(4):443-453; quiz 454.

DOI: https://doi.org/10.1007/s00482-012-1202-0 
Schmerz 2012 $\cdot 26: 443-454$

DOI 10.1007/s00482-012-1202-0

(c) Deutsche Schmerzgesellschaft e.V. Published by Springer-Verlag -

all rights reserved 2012

\section{Redaktion}

H. Göbel, Kiel

R. Sabatowski, Dresden

\section{\begin{tabular}{|l|}
\hline Punkte sammeln auf... \\
\hline springermedizin.de/
\end{tabular} eAkademie \\ Teilnahmemöglichkeiten \\ Diese Fortbildungseinheit steht Ihnen als e.CME und e.Tutorial in der Springer Medizin e.Akademie zur Verfügung. \\ - e.CME: kostenfreie Teilnahme im Rahmen des jeweiligen Zeitschriften- abonnements \\ - e.Tutorial:Teilnahme im Rahmen des e.Med-Abonnements}

\section{Zertifizierung}

Diese Fortbildungseinheit ist mit 3 CMEPunkten zertifiziert von der Landesärztekammer Hessen und der Nordrheinischen Akademie für Ärztliche Fort- und Weiterbildung und damit auch für andere Ärztekammern anerkennungsfähig.

\section{Hinweis für Leser aus Österreich} Gemäß dem Diplom-Fortbildungs-Programm (DFP) der Österreichischen Ärztekammer werden die in der e.Akademie erworbenen CME-Punkte hierfür 1:1 als fachspezifische Fortbildung anerkannt.

\section{Kontakt und weitere}

Informationen

Springer-Verlag GmbH

Springer Medizin Kundenservice

Tel. 08007780777

E-Mail:kundenservice@springermedizin.de

\section{CME Zertifizierte Fortbildung}

\author{
J. Mauch · M. Weiss
}

Anästhesieabteilung, Universitäts-Kinderkliniken Zürich

\section{Kaudalanästhesie bei Kindern}

\section{Stellenwert und Aspekte zur Sicherheit}

\section{Zusammenfassung}

Der Kaudalblock ist die am häufigsten durchgeführte Regionalanästhesietechnik zur perioperativen Analgesie bei Kindern. Komplikationen treten äußerst selten auf. Dennoch sind bei der Indikationsstellung lokale und systemische Ausschlusskriterien zu prüfen. Zur Gewährleistung einer optimalen Qualität und Sicherheit sind technische Details strikt zu beachten. Intravaskuläre Injektionen sind unbedingt zu vermeiden. Zu deren Früherkennung ist der Adrenalinzusatz hilfreich. Die Wahl des Lokalanästhetikums (LA) hingegen dürfte hinsichtlich der Sicherheit von zweitrangiger Bedeutung sein. Als Adjuvans bietet Clonidin bei geringen Nebeneffekten die meisten Vorteile. Die systemische LA-Intoxikation ist eine sehr seltene, aber potenziell letale Komplikation. Entsprechend müssen alle Anstrengungen zu Prävention und Früherkennung unternommen werden. Kommt es trotzdem zum Herz-Kreislauf-Kollaps, werden unverzüglich Reanimationsmaßnahmen gemäß den aktuellen Leitlinien eingeleitet. Adrenalin ist dabei Erstlinienmedikation; die Lipidtherapie ist kein Ersatz, sondern eine mögliche sekundäre Ergänzung.

\section{Schlüsselwörter}

Lokalanästhetika · Adrenalin · Elektrokardiogramm · Blutdruck · Toxizität 


\section{Lernziel}

Nach Lektüre dieses Beitrags

- wissen Sie, welche Voraussetzungen für die Durchführung eines Kaudalblocks (KB) erfüllt sein müssen,

- überblicken Sie die technischen Details, die es dabei zu beachten gilt,

- kennen Sie die Lokalanästhetika (LA) und Adjuvanzien, die beim KB zum Einsatz kommen,

sind Sie mit den Maßnahmen zur Vermeidung bzw. zur frühen Erkennung und Therapie einer systemischen LA-Intoxikation vertraut.

\section{Einleitung}

Die Kombination von Allgemein- und Regionalanästhesie ist ein Grundpfeiler der modernen Kinderanästhesie. Sie vermindert Stressreaktionen, erlaubt eine Reduktion der Anästhetika und teilweise sogar einen Verzicht auf Opiate, wodurch postoperativ die Gefahr der Atemdepression sowie die Inzidenz von Übelkeit und Erbrechen reduziert werden. Die optimale und über Stunden anhaltende Analgesie gewährt ein ruhiges Erwachen und erhöht die Zufriedenheit von Patient, Eltern und medizinischem Personal. Bei richtiger Anwendung ist der Kaudalblock (KB) das optimale Regionalanästhesieverfahren zur perioperativen Analgesie bei vielen Eingriffen in der Kinderchirurgie.

Trotz des zunehmenden Trends zu peripheren Nervenblockaden [1] ist der KB die am häufigsten durchgeführte Regionalanästhesietechnik zur perioperativen Analgesie in der Kinderanästhesie. Er bietet eine zuverlässige Analgesie für alle Operationen der unteren Körperhälfte bis zum Nabel. Der $\mathrm{KB}$ ist einfach durchzuführen, entsprechend rasch erlernbar und eine sichere Technik [1, 2, 3]. Besondere Sorgfalt ist jedoch bei kleinen Säuglingen von Nöten, bei denen die korrekte Punktion des Hiatus sacralis gelegentlich schwieriger ist und Komplikationen häufiger sind [1, 3, 4].

\section{Voraussetzungen}

Sowohl inspektorisch als auch anamnestisch dürfen keine Hinweise auf pathologisch-anatomische Veränderungen des lumbosakralen Spinalkanals vorliegen, z. B. eine Meningomyelozele. Da diverse seltene Syndrome mit Anomalien im Bereich der Wirbelsäule einhergehen können, sollte im individuellen Fall die aktuelle Referenzliteratur konsultiert werden. Lokale Infekte oder eine schwere

\section{Pediatric caudal anesthesia $\cdot$ Importance and aspects of safety concerns}

Lokale Infekte oder eine schwere Sepsis sind absolute Kontraindikationen für einen Kaudalblock

\begin{abstract}
Caudal block is a safe procedure commonly used for pediatric perioperative analgesia. Complications are extremely rare but nevertheless local and systemic contraindications must be excluded. Optimal safety and quality result when strict attention is paid to technical details. A local anesthetic (LA) containing epinephrine allows early detection of inadvertent intravascular LA administration; therefore an epinephrine/LA mixture is recommended at least for the test dose. In terms of safety the choice of LA itself is probably of secondary importance. Clonidine as an adjuvant has an excellent risk/benefit profile with minimal side effects. Inadvertent systemic LA intoxication is a rare but potentially fatal complication of regional anesthesia and measures for prevention and early detection are essential. Should circulatory arrest occur, immediate resuscitation following standard guidelines is to be initiated including the use of epinephrine as the first line drug. Intravenous administration of lipid solutions may be beneficial as a secondary adjunct to stabilize hemodynamics but is not an alternative to epinephrine.
\end{abstract}

\section{Keywords}

Anesthetics, local · Adrenaline $\cdot$ Electrocardiogram $\cdot$ Blood pressure $\cdot$ Toxicity 
Abb. 1 Anatomische Landmarken für die Punktion des Hiatus sacralis am Skelett. Die Spinae iliacae posteriores superiores bilden mit dem Hiatus sacralis ein gleichseitiges Dreieck

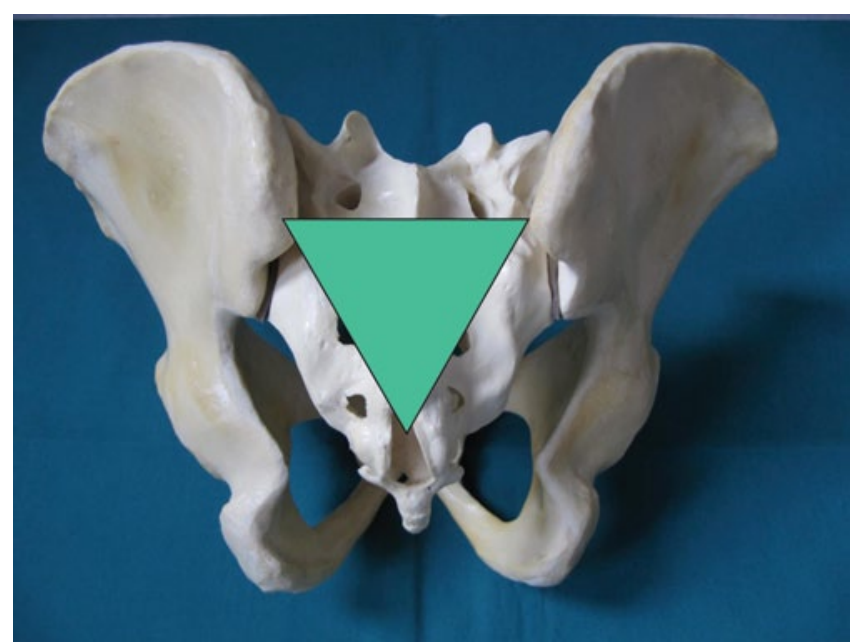

Sepsis stellen absolute Kontraindikationen für einen KB dar [5], dies gilt ebenso für eine Meningitis. Eine Allergie auf Lokalanästhetika (LA) verbietet eine Regionalanästhesie. Die Ablehnung der Technik durch die Eltern oder das Kind muss respektiert werden. Insbesondere Kinder mit einer Behinderung profitieren von der optimalen Schmerzkontrolle mithilfe des KB. Dieser Aspekt sollte bei Vorliegen eines aktiven neurologischen Leidens in die Nutzen-Risiko-Analyse einfließen. Auch bei Kindern mit ventrikuloperitonealem Shunt muss der Nutzen eines KB gegen die zusätzlichen Risiken individuell abgewogen werden. Dabei muss v. a. die Drucksteigerung im Spinalkanal berücksichtigt werden, die durch die LA-Injektion verursacht wird [6].

Die anatomischen Landmarken für die Punktion am Skelett sind in • Abb. 1 dargestellt. Bei palpatorischer Unsicherheit hinsichtlich der ossären Strukturen kann der Hiatus sacralis sonographisch lokalisiert werden. Ansonsten wird der Ultraschall für die Kaudalpunktion nicht routinemäßig benötigt. Zur Supervision und zur Visualisierung der Ausbreitung des injizierten LA und damit der erfolgreichen Punktion des Epiduralraums kann die ultraschallgestützte Technik nützlich sein.

Bei unauffälliger standardisierter Gerinnungsanamnese des Kindes und der Eltern sollte auf eine laborchemische Analyse der Blutgerinnung vor Anlage eines KB verzichtet werden [5]. Bei Frühgeborenen ist darauf zu achten, dass Vitamin K (Konakion ${ }^{\circledR}$ ) im üblichen Schema appliziert wurde. An der Institution der Autoren wird die Gabe von Konakion ${ }^{\varpi} 48-72$ h vor einer elektiven Herniotomie in wacher Kaudalanästhesie bzw. Allgemeinanästhesie mit KB wiederholt. Grundsätzlich werden für die Anlage eines KB von den Autoren:

- ein Quick-Wert $\geq 50 \%$,

- eine aktivierte partielle Thromboplastinzeit (aPTT) $\leq 50 \mathrm{~s}$ und

- eine Thrombozytenzahl $\geq 50 \bullet 10^{9} / 1$

gefordert. Bei auffälliger Anamnese muss eine gezielte Abklärung erfolgen. Je nach Ausprägung der Gerinnungsstörung müssen individuell Nutzen und Risiko evaluiert werden. Das Blutungsrisiko beim KB ist aus anatomischen und punktionstechnischen Gründen nicht gleich hoch anzusetzen wie bei den anderen zentralen Blockadetechniken. Vermutlich ist es eher den peripheren Nervenblockaden vergleichbar. In der Literatur wurde bislang kein epidurales Hämatom nach Anlage eines KB beschrieben. Eine Herz-Kreislauf-Überwachung mit nichtinvasiver, 1-minütlicher Blutdruckmessung und kontinuierlichem Elektrokardiogramm, in dem P-Welle, QRS-Komplex und T-Welle gut sichtbar dargestellt werden, ist eine obligatorische Voraussetzung bei der Anlage eines KB - wie auch bei jedem anderen regionalanästhetischen Verfahren. Insbesondere bei Adoleszenten werden T-Wellenabnormitäten mit der EKG-Ableitung II sensitiver erkannt als mit EKG-Ableitung I [7].

\section{Material}

Eine Umfrage in der Association of Paediatric Anaesthetists of Great Britain and Ireland (APAGBI, [8]) ergab, dass von den Durchführenden für die Punktion eine Venenkanüle (69,7\%) favorisiert wird, gefolgt von Hohlnadeln (16,2\%) und Nadeln mit Stilett (14,1\%). Eine mögliche Verschleppung
Der Ultraschall wird für die Kaudalpunktion nicht routinemäßig benötigt

Die Herz-Kreislauf-Überwachung ist eine obligatorische Voraussetzung bei der Anlage eines Kaudalblocks

Für die Punktion wird eine Venenkanüle favorisiert 
Mit einer Plastikkanüle sind die Duraperforation und die Penetration ins Periost kaum wahrscheinlich

Es muss auf streng aseptisches Arbeiten geachtet werden

Beim schlafenden Kind hat sich zur Punktierung die Seitenlage bewährt

Nach Perforation des Lig. sacrococcygeum sollte die Nadel maximal 1-2 $\mathrm{mm}$ vorgeschoben werden von epidermalem Gewebe wurde mehrheitlich (91,5\%) als nichtwahrscheinlich bezeichnet. Entsprechend verzichten 87,2\% der Befragten auf eine Hautperforation vor der Punktion. Baris et al. [9] untersuchten Hohl- und Kaudalnadeln mit Stilett nach kaudaler Punktion auf zelluläres Material. In keiner Probe konnten Zellen mit mitotischer Aktivität aus dem Stratum basale gefunden werden. Zellen ohne mitotische Aktivität wurden in beiden Gruppen in 8,5\% der Fälle identifiziert. Die beiden Nadeltypen unterschieden sich nicht hinsichtlich des potenziellen Transports von epidermalen Zellen. Es gibt keinen Fallbericht, in dem über einen Epidermoidtumor im Zusammenhang mit einer vorhergehenden Kaudalanästhesie berichtet wird. Hinsichtlich der Nadelgröße wurden 22-GModelle am häufigsten (68,6\%) verwendet, gefolgt von 20-G- (17,6\%), 24-G- (10,5\%) und 18-G-Varianten $(3,2 \%,[8])$.

In der Institution der Autoren wird der KB seit vielen Jahren mit gutem Resultat und ohne lokale Komplikationen mithilfe einer Venenkanüle ohne vorherige Hautperforation gesetzt. Nach Perforation des Lig. sacrococcygeum wird die Plastikkanüle vorsichtig in den Periduralraum vorgeschoben. Dort erfolgt dies typischerweise ohne jeglichen Widerstand. Der Einsatz einer Plastikkanüle weist gegenüber einer einfachen Nadel oder Nadel mit Stilett entscheidende Vorteile auf: Mit der Entfernung der Punktionsnadel aus der Kanüle entfällt das Risiko der Verschleppung von epidermalem Gewebe in den Epiduralraum. Mit einer Plastikkanüle sind die Duraperforation und die Penetration ins Periost kaum wahrscheinlich, die Punktion einer Kaudalvene seltener und die Dislokation der Plastikkanüle während der LA-Injektion (vgl. Spritzenwechsel) eher unwahrscheinlich. Letzteres ist besonders wichtig, wenn der KB beim ehemals frühgeborenen wachen, unruhigen Patienten als alleinige Regionalanästhesie gesetzt wird. Für Kinder $\leq 10 \mathrm{kgKG}$ bzw. bis zum Alter von 1 Jahr werden 24-G-, anschließend 22-G-Kanülen verwendet. Ab einem Patientenalter von 8-10 Jahren kommen 20-G-Kanülen zum Einsatz.

\section{Durchführung}

\section{Sterilität}

Auch wenn lediglich eine Einmalinjektion vorgesehen und in der Literatur nur 1 Fall einer schweren lokalen Infektion nach KB beschrieben ist [10], muss auf streng aseptisches Arbeiten geachtet werden. Die Einwirkzeit des entsprechenden Desinfektionsmittels muss zwingend abgewartet werden. Ein alkoholhaltiges Desinfektionsmittel ist zu empfehlen, da die Einwirkzeit lediglich 1 min beträgt $[11,12]$. Das Tragen von sterilen Handschuhen ist Standard $[8,13]$.

\section{Lagerung}

Die Lagerung des Patienten muss im Wesentlichen 2 Kriterien erfüllen:

- Der Hiatus sacralis muss für den Anästhesisten bequem punktierbar sein.

- Der Patient sollte sich während Punktion und LA-Injektion nicht aus dieser Lage heraus bewegen.

Beim schlafenden Kind hat sich die Seitenlage ( $\bullet$ Abb. 2a) bewährt: Linksseitenlage für rechtshändig punktierende Anästhesisten und vice versa. Wird der KB beim kleinen wachen Säugling als alleinige Regionalanästhesie angelegt, empfiehlt sich die Punktion in der Froschstellung (• Abb. 2b). Dabei kann das wache Kind gut gegen die Unterlage fixiert und optimal stabilisiert werden. In Bezug auf Besonderheiten der Wachkaudalanästhesie wird auf die Arbeiten von Gerber u. Weiss [14] sowie Hölzle et al. [15] verwiesen.

\section{Punktion}

Die palpatorische Lokalisation des Hiatus sacralis unter Berücksichtigung der ossären Landmarken (- Abb. 1) ist Standard. Der Ultraschall wird, wie erwähnt, selten [8] und in unklaren Situationen eingesetzt $[16,17]$. Nach Perforation des Lig. sacrococcygeum sollte die Nadel maximal 1-2 mm vorgeschoben werden. Dies ist insbesondere bei kleinen Säuglingen streng zu beachten, denn bei der Geburt reicht der Duralsack bis auf Höhe von S4, mit 1 Jahr bis S2. Wird die Dura akzidentell perforiert, kann eine totale Spinalanästhesie resultieren. Die Häufigkeit einer akzidentellen Duraperforation 

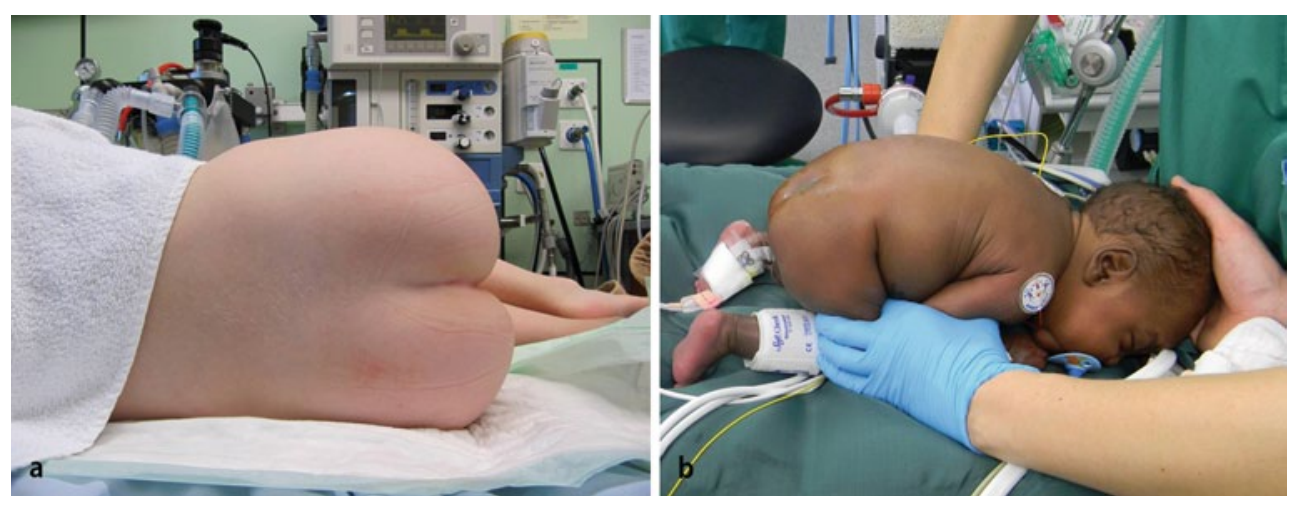

Abb. $2 \Delta$ Lagerung des Patienten zur Anlage des Kaudalblocks. a Seitenlage beim anästhesierten Kind; b Froschstellung beim wachen Säugling

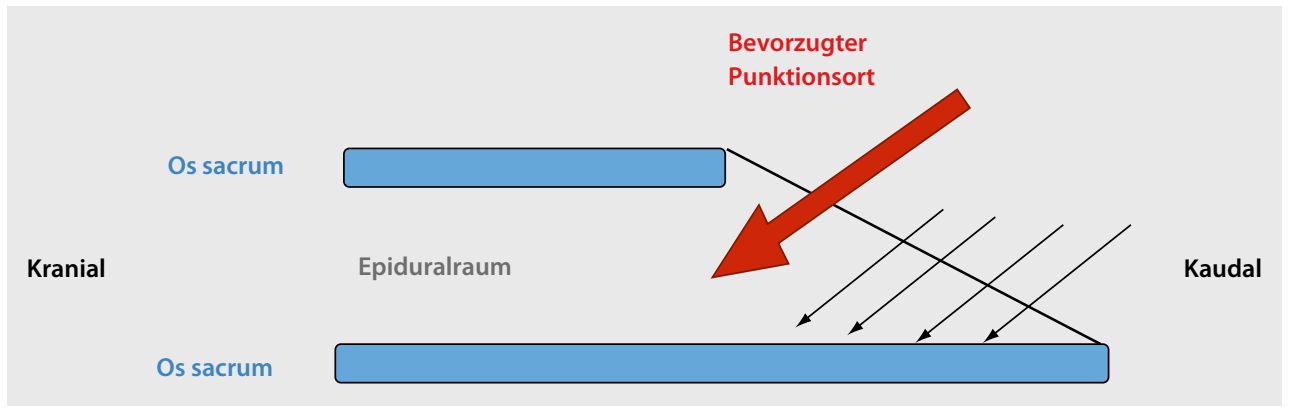

Abb. $3 \Delta$ Schematischer Sagittalschnitt durch den Hiatus sacralis. Der Epiduralraum ist am kranialen Rand des Hiatus sacralis am tiefsten und deutlich einfacher zu punktieren

wird in der Literatur mit 4:15.013 [3] und mit 6:8493 [1] angegeben. In der Arbeit von Ecoffey et al. [1] waren ausschließlich Säuglinge betroffen. Postpunktionelle Kopfschmerzen wurden nicht beobachtet. Bei kleinen Säuglingen ist das Sakrum verhältnismäßig weich und mit einer Nadel leicht zu penetrieren. Wird das LA intraossär appliziert, werden rasch hohe Plasmaspiegel erreicht, die Symptome und Zeichen einer systemischen Intoxikation verursachen können.

Gelingt die Punktion des Hiatus sacralis nicht wie gewünscht, sind die beiden folgenden häufig begangenen Fehler zu überprüfen bzw. zu korrigieren:

- Der Anästhesist hat sich in der Beurteilung der Mittellinie von der Rima ani fehlleiten lassen. In Seitenlage verschiebt sich die Rima ani, abhängig von Volumen und Beschaffenheit der Weichteile, vertikal bodenwärts.

- Die Punktion erfolgt im kaudalen Anteil des Hiatus sacralis. Hier ist die Distanz zwischen Lig. sacrococcygeum und Sakrumperiost sehr kurz. Nach Penetration des Ligaments steht die Nadel unmittelbar am Periost an und es ist schwierig, den epiduralen Raum ausfindig zu machen. Letzterer ist am kranialen Rand des Hiatus sacralis am größten und deutlich einfacher zu punktieren (• Abb. 3).

\section{Injektion}

Vor und während der LA-Injektion sollte die Nadel bzw. Kanüle auf einen passiven Rückfluss von Blut (allenfalls von Liquor) hin geprüft und ein Aspirationstest durchgeführt werden, auch wenn damit die intravaskuläre Lage in 57\% der Fälle nicht erkannt wird [18]. Die Injektion sollte langsam, über mindestens 1 min, erfolgen, um [19]:

- akute, exzessive Drucksteigerungen im Epidural-/Duralraum zu vermeiden,

- bei akzidenteller intravaskulärer Lage hohe LA-Plasmaspitzenspiegel zu vermeiden und

- eine systemische Intoxikation frühzeitig zu erkennen.
Bei kleinen Säuglingen ist das Sakrum mit einer Nadel leicht zu penetrieren

Die Injektion sollte langsam erfolgen 
Nach Entfernen der Kanüle wird die Einstichstelle mit einem minimal auftragenden Klebeverband abgedeckt.

Der „Whoosh-Test“ [20] ist nicht zu empfehlen [21], da die dabei durchgeführte epidurale Injektion von Luft zu einer qualitativ schlechten, fleckförmigen Anästhesie führen kann. Bei Lachgaseinsatz können epidurale Gasblasen entstehen. In der Literatur sind sogar venöse Luftembolien beschrieben [22].

\section{Lokalanästhetikum}

Eine möglichst gute und lang andauernde Analgesie wird angestrebt

Bupivacain zeigt die höchste Inzidenz motorischer Blockaden

Bei Säuglingen ist die Bindungskapazität für Lokalanästhetika im Plasma reduziert
Mit dem KB sollen nicht nur intraoperativ, sondern v. a. postoperativ Opiate eingespart werden. Zudem wird eine möglichst gute und lang andauernde Analgesie angestrebt. Aus diesen Gründen werden die lang wirksamen LA Bupivacain, Levobupivacain und Ropivacain eingesetzt. Welche Subs$\operatorname{tanz}$ für den KB präferenziell verwendet wird, ist regional sehr unterschiedlich. Eine Umfrage unter den APAGBI-Kinderanästhesisten [8] ergab vergleichbare Einsatzhäufigkeiten für razemisches Bupivacain $(43,4 \%)$ und Levobupivacain $(41,7 \%)$, während Ropivacain $(13,4 \%)$ deutlich seltener verwendet wurde. Im Unterschied dazu kam im französischsprachigen Raum [1] in 85\% aller Regionalanästhesien Ropivacain zum Einsatz.

Eine Literaturübersicht [23] zum LA mit der besten Wirksamkeit und den geringsten Nebeneffekten für den KB ergab, dass die 3 Substanzen hinsichtlich der Wirksamkeit, d. h. der Anschlagzeit, intraoperativen Qualität und postoperativen Analgesiedauer, ebenbürtig sind. Nebeneffekte betreffend zeigte Bupivacain die höchste Inzidenz motorischer Blockaden und Ropivacain die tiefste Blockade. Intraoperative selbstlimitierende hämodynamische Veränderungen sowie postoperative Übelkeit und Erbrechen traten unabhängig vom verwendeten LA auf. Schwere Nebenwirkungen wie kardiale und neurologische Toxizität wurden in den betrachteten randomisierten, kontrollierten Studien (bis August 2010) nicht beschrieben. In Tierexperimenten wurde hingegen gezeigt, dass Ropivacain und Levobupivacain verglichen mit razemischem Bupivacain im Provokationstest das bessere Risikoprofil aufweisen [24, 25]. In der täglichen Praxis wird für den KB in Kombination mit einer Allgemeinanästhesie meist 0,125\%iges razemisches Bupivacain oder 0,2\%iges Ropivacain verwendet. Für eine Analgesie der sakralen Segmente werden 0,5-0,7 ml/kgKG, für lumbale und sakrale Segmente 1-1,2 ml/kgKG und für den zusätzlichen Einschluss tief- bis mittelthorakaler Segmente $1,5 \mathrm{ml} / \mathrm{kgKG}$ benötigt [26]. In der Regel wird das LA-Gesamtvolumen $<30 \mathrm{ml}$ betragen. In speziellen Situationen können - unter Berücksichtigung der empfohlenen LA-Maximaldosis - bis maximal $40 \mathrm{ml}$ appliziert werden.

\section{Systemische Intoxikation mit dem Lokalanästhetikum}

\section{Allgemeines}

Das Risiko einer systemischen LA-Intoxikation dürfte bei kleinen Kindern höher sein als bei Erwachsenen. Einerseits werden in Relation zum Körpergewicht hohe LA-Dosen appliziert. Die kleinen absoluten Volumina können mit den entsprechend kleineren Spritzen auch mit höherem Druck bzw. schneller verabreicht werden; dies kann schneller zu ausgeprägteren Nebenwirkungen führen. Weiterhin sind bei Säuglingen die Plasmaspiegel des sauren $\alpha_{1}$-Glykoproteins niedriger, damit ist die Bindungskapazität für LA reduziert. Inwieweit dies jedoch klinisch von Bedeutung ist, ist nicht ganz klar. Insgesamt ist die systemische LA-Intoxikation ein seltenes Ereignis. Große Studien berichten über 8 Fälle bei 158.229 KB [2], 3 Fälle bei 15.013 KB [3] und 7 Fälle bei 8493 KB [1]; der Schweregrad der Intoxikation wird nicht beschrieben. Ein permanenter Schaden trat nicht auf. Es sind 2 Fälle mit Herz-Kreislauf-Stillstand nach KB und erfolgreicher Reanimation beschrieben [27, 28]. In einem Fall [27] wurde akzidentell 1\%iges Ropivacain anstelle der vorgesehenen 0,2\%igen Lösung appliziert, woraus eine 5-fache Überdosierung resultierte. Im anderen Fall [28] wird eine unbemerkte systemische Injektion als Ursache für den Herz-Kreislauf-Kollaps vermutet.

Im Gegensatz zu Erwachsenen werden bei Kindern LA in der Regel in tiefer Sedierung oder Allgemeinanästhesie appliziert. Unter diesen Umständen fehlen bei akzidenteller intravaskulärer Injektion die neurologischen Frühwarnzeichen einer systemischen Intoxikation wie:

- Unruhe,

- Tinnitus, 
- Sehstörungen und

- verwaschene Sprache.

Beim relaxierten Kind ist ein Krampfanfall nicht sichtbar. Die kardiovaskuläre Depression mit HerzKreislauf-Kollaps kann ohne andere Symptome oder Zeichen unvermittelt auftreten. Ursächlich dürfte in den meisten Fällen eine unbemerkte intravasale LA-Injektion oder ein Dosierungsfehler sein (Verwechslung in der Konzentration des LA, [1, 27]). Zeichen der zunehmenden kardialen Toxizität sind:

- Bradykardie,

- Hypotonie,

- verbreiterte QRS-Komplexe und

- hohe T-Wellen.

Sie können dem Herz-Kreislauf-Kollaps vorausgehen [27, 29].

\section{Maßnahmen zur Verhinderung}

Primär muss sichergestellt werden, dass das richtige Medikament in der richtigen Konzentration und Menge vorbereitet ist. Insbesondere die Konzentration muss genau kontrolliert werden, da für eine bestimmte Substanz in verschiedenen Konzentrationen oft ähnliche Verpackungen und Aufkleber für die Spritzenbeschriftung gewählt werden. Ein Irrtum in der Konzentration kann fatale Folgen haben [27]. Die zu injizierende Menge wird anhand der Größe bzw. des Gewichts des Kindes und der gewünschten Ausbreitung der Analgesie individuell berechnet. Hier erhöht das Vieraugenprinzip die Kontrolle der zu applizierenden Menge durch eine zweite Person - die Sicherheit. Die empfohlenen Höchstdosen sollten berücksichtigt werden [5]:

- $2,5 \mathrm{mg} / \mathrm{kgKG}$ für Bupivacain und

- $3 \mathrm{mg} / \mathrm{kgKG}$ für Ropivacain.

Während der LA-Applikation müssen verschiedene Punkte beachtet werden. Die American Society of Regional Anesthesia and Pain Medicine (ASRA) weist darauf hin, dass es keine einzelne Maßnahme gibt, die eine systemische LA-Intoxikation mit Sicherheit verhindert. Infolgedessen empfiehlt sie, parallel verschiedene Aspekte zu beachten, die die Sicherheit der Regionalanästhesie erhöhen (- Tab. 1). Erstaunlich ist die Diskrepanz zwischen hohem Empfehlungsgrad und niedrigem Evidenzlevel. Untersuchungen an Babyschweinen konnten jedoch kürzlich belegen, dass eine schnelle Injektionsgeschwindigkeit, wenn sie akzidentell intravasal erfolgt, zu überproportional hohen Plasmaspiegeln und zu einem unmittelbaren Herz-Kreislauf-Kollaps führt. Bei einer langsamen Injektionsgeschwindigkeit hingegen wird der Anästhesist, wenn akzidentell intravasal appliziert wird, durch eine langsam progrediente Herz-Kreislauf-Depression bereits bei einer niedrigeren verabreichten Dosis auf die Problematik aufmerksam und kann die LA-Injektion stoppen [19]. Diese Resultate belegen eindeutig den Nutzen bzw. die Wichtigkeit der langsamen Injektionstechnik.

Eine akzidentelle intravasale LA-Applikation muss so rasch wie möglich erkannt und gestoppt werden. Die adrenalinhaltige Testdosis zur frühzeitigen Erkennung einer intravasalen Injektion wird in der Literatur kontrovers diskutiert und in der Praxis nicht konsequent verwendet. In der pädiatrischen Anästhesie sollte die Testdosis 0,5-1 $\mu \mathrm{g} / \mathrm{kgKG}$ Adrenalin (maximal $15 \mu \mathrm{g}$ ) enthalten. Die hämodynamische Reaktion variiert je nach verwendetem Anästhetikum (Halothan, Isofluran, Sevofluran, Propofol) und ist davon abhängig, ob zuvor Atropin verabreicht wurde. Verglichen mit dem kardiovaskulären Steady State in Narkose, gelten als Schwellenwerte für eine positive Reaktion [31]: - eine Zunahme der Herzfrequenz um $\geq 10$ Schläge/min,

- eine Zunahme des systolischen Blutdrucks um $\geq 15 \mathrm{mmHg}$ und

- eine Zunahme der T-Welle im EKG um $\geq 25 \%$ des Ausgangswerts.

Bei der Anlage der Regionalanästhesie muss eine adäquate Narkosetiefe gewährleistet sein, da ansonsten die durch die Punktion ausgelöste Schmerzreaktion einen positiven Testdosiseffekt vortäuschen kann. Wird das Kind unbeabsichtigt stimuliert, muss mit der Injektion der Testdosis bis zum erneuten Erreichen der Steady-State-Bedingungen gewartet werden. Eine kürzlich durchgeführte Untersuchung an Kindern im Alter von 1 Monat bis 16 Jahren in Sevoflurannarkose [7] zeigte, dass

Insbesondere die Konzentration des Medikaments muss genau kontrolliert werden

Keine einzelne Maßnahme kann eine systemische Intoxikation mit dem Lokalanästhetikum mit Sicherheit verhindern

Eine akzidentelle intravasale LA-Applikation muss so rasch wie möglich erkannt und gestoppt werden

Bei der Anlage der Regionalanästhesie muss eine adäquate Narkosetiefe gewährleistet sein 
Tab. 1 Maßnahmen zur Prävention einer systemischen LA-Intoxikation. (Adaptiert nach [30])

\begin{tabular}{lll}
\hline Maßnahme & Empfehlungsklasse & $\begin{array}{l}\text { Evidenz- } \\
\text { grad }\end{array}$ \\
\hline Niedrigste effektive LA-Dosis applizieren & $\mathrm{I}$ & $\mathrm{C}$ \\
\hline $\begin{array}{l}\text { Gesamtdosis in mehreren kleinen Teildosen mit intermittierenden Pausen } \\
\text { von 15-30 s verabreichen }\end{array}$ & $\mathrm{I}$ & $\mathrm{C}$ \\
\hline Aspirationsprobe mit Nadel oder Katheter vor LA-Injektion durchführen & $\mathrm{I}$ & $\mathrm{C}$ \\
\hline $\begin{array}{l}\text { Bei Verabreichung von potenziell toxischen LA-Dosen wird der Zusatz } \\
\text { eines intravasalen Markers (Adrenalin) empfohlen. }\end{array}$ & Ila & $\mathrm{B}$ \\
\hline $\begin{array}{l}\text { Eine LA-Injektion unter Ultraschallkontrolle kann möglicherweise die } \\
\text { Häufigkeit einer intravasalen Injektion reduzieren. }\end{array}$ & Ila \\
\hline LA Lokalanästhetikum. & & $\mathrm{C}$ \\
\hline
\end{tabular}

eine i.v.-injizierte Testdosis von Bupivacain mit Adrenalin ( $1 \mu \mathrm{g} / \mathrm{kgKG}$, maximal $15 \mu \mathrm{g})$ in 100\% der Fälle erkannt wird, wenn das EKG (Herzfrequenz und T-Welle) über 1 min beobachtet und der Blutdruck 1 min nach Injektion nichtinvasiv gemessen wird. Neben der Zunahme der Herzfrequenz muss auch die Abnahme um $\geq 10$ Schläge/min als positives Zeichen gewertet werden. Möglicherweise handelt es sich dabei um eine Reflexbradykardie, ausgelöst durch den Blutdruckanstieg. Die Veränderungen von Herzfrequenz, T-Welle und Blutdruck sind altersabhängig. Bei kleinen Kindern tritt die T-Wellenerhöhung äußerst zuverlässig auf, bei Adoleszenten hingegen nicht. Bei Letzteren kommt es häufig zu einer Reduktion der T-Welle oder zu einem biphasischen Verlauf (• Abb. 4). Die Veränderung der Herzfrequenz (insbesondere die Abnahme) und der Anstieg des systolischen Blutdrucks 1 min nach Injektion sind bei Adoleszenten die deutlich sensitiveren Parameter. Ob die hohe Aussagekraft und Zuverlässigkeit der Testdosis bei Kindern in Sevoflurananästhesie auch während einer i.v.-Anästhesie mit Propofol gegeben ist, bleibt noch zu klären [32].

Ob die T-Wellenerhöhung im EKG durch den Adrenalinzusatz oder durch das LA selbst zustande kommt, wurde in der Vergangenheit kontrovers diskutiert und hat zu etlichen Verwirrungen geführt. Differenzierte Untersuchungen an Schweinen haben gezeigt, dass Adrenalin niedrig dosiert, beispielsweise als Testdosiszusatz mit $1 \mu \mathrm{g} / \mathrm{kgKG}$, T-Wellenveränderungen bewirkt. Bupivacain allein kann, wenn hohe Dosen i.v. verabreicht werden, ebenfalls T-Wellenerhöhungen verursachen [29]. Dies darf jedoch nicht als Frühwarnzeichen einer systemischen Intoxikation gewertet werden, sondern ist bereits Zeichen der kardialen Toxizität mit drohendem Herz-Kreislauf-Kollaps. Mit anderen Worten: Wenn durch Bupivacain hervorgerufene T-Wellenerhöhungen detektiert werden, ist es eigentlich schon zu spät. Für die Früherkennung einer intravasalen Injektion erforderlich sind:

- der Adrenalinzusatz,

- eine kontinuierliche EKG-Überwachung und

- Blutdruckmessungen in 1-minütlichen Abständen.

\section{Therapie}

Die systemische LA-Intoxikation ist eine schwerwiegende Komplikation mit potenziell letalem Ausgang. Entsprechend müssen alle Anstrengungen zu Prävention und Früherkennung unternommen werden. Kommt es trotzdem zum Herz-Kreislauf-Kollaps, muss unverzüglich gemäß den aktuellen Leitlinien reanimiert werden. Als zusätzliche Maßnahme bei systemischer LA-Intoxikation wird seit einigen Jahren die Therapie mit Intralipid ${ }^{\circledR}$, die „lipid rescue“, propagiert (http://www.lipidrescue.org). Das Konzept hat große Akzeptanz und durch die Aufnahme in Leitlinien weite Verbreitung gefunden, obwohl vieles nicht klar ist [33]. In diversen Kasuistiken - einschließlich pädiatrischer Fälle - wurde der erfolgreiche Einsatz beschrieben. Relevante Nebenwirkungen sind bis jetzt nicht dokumentiert. Vereinzelt wurde Intralipid ${ }^{\circledR}$ sogar als Erstlinientherapie bei systemischer Intoxikation vorgeschlagen [34, 35]. Untersuchungen an Babyschweinen belegten jedoch, dass sowohl bei schwerer Herz-Kreislauf-Depression infolge einer LA-Intoxikation als auch beim Herz-Kreislauf-Stillstand Adrenalin als Erstlinientherapie deutlich effektiver als Intralipid ${ }^{\circ}$ ist und somit unverzichtbar bleibt [36, 37]. Entsprechend muss bei einer LA-Intoxikation mit Herz-Kreislauf-Depression oder -kollaps weiterhin primär nach den klassischen Algorithmen reanimiert und Intralipid ${ }^{\circledR}$ in zweiter Linie eingesetzt werden.

Tritt beim wachen Patienten LA-induziert ein generalisierter Krampfanfall auf, kann die Therapie mit Propofol effektiv sein. Verantwortlich dafür ist wahrscheinlich die antikonvulsive Eigenschaft 
I

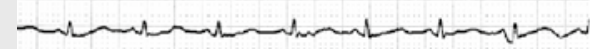

11

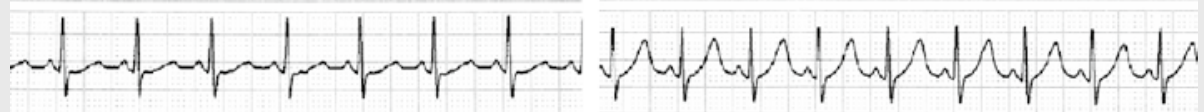

a

I

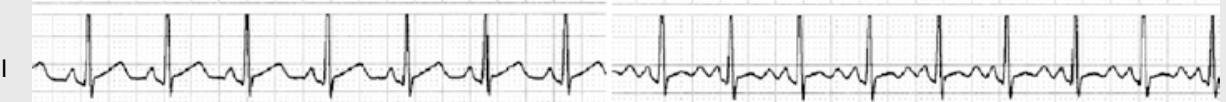

b

Abb. $4 \Delta$ EKG-Ableitungen I und II vor (links) und nach (rechts) i.v.-Injektion einer Testdosis von 0,125\%igem Bupivacain mit Adrenalin 1:200.000. a Ein 5,3-jähriges Mädchen, b ein 15-jähriges Mädchen

von Propofol und nicht der Lipidanteil in der Formulierung. Auf keinen Fall darf Propofol als Intralipid $^{\oplus}$-Ersatz beim LA-induzierten Herz-Kreislauf-Kollaps verwendet werden. Cave: Propofol ist in dieser Situation aufgrund seiner kardiodepressiven Eigenschaften absolut kontraindiziert.

\section{Adjuvanzien zum Lokalanästhetikum}

Um die Wirkung des LA bzw. die Analgesie des KB zu verlängern und/oder zu verstärken, werden immer wieder medikamentöse Zusätze getestet. Dadurch können die Gesamtdosis des LA und dosisabhängige Nebenwirkungen reduziert werden. Insbesondere durch Reduktion der LA-Konzentration treten weniger motorische Blockaden auf, was die Frühmobilisation ermöglicht und das Wohlbefinden der Kinder steigert. Die folgenden Abschnitte geben eine Übersicht über die meistdiskutierten Adjuvanzien.

Adrenalin. Adrenalin führt nur zu einer unwesentlichen Wirkungsverlängerung des LA. In einer Konzentration von $5 \mu \mathrm{g} / \mathrm{ml}$ wird es hauptsächlich als Testdosis verwendet $(0,1-0,2 \mathrm{ml} / \mathrm{kgKG}$, maximal $15 \mu \mathrm{g}$ ). Durch die vasokonstriktorischen Eigenschaften könnte theoretisch lokal die Perfusion des Rückenmarks kompromittiert werden, bisher hat sich dies aber nicht klinisch manifestiert. Untersuchungen an Kaninchen mit epidural verabreichtem 2\%igem Lidocain+Adrenalin ergaben keine Reduktion des spinalen Blutflusses. Jedoch war die Aufrechterhaltung eines adäquaten systemischen Blutdrucks für die spinale Perfusion relevant [38].

Clonidin. Clonidin wird in einer Dosierung von 1-2 $\mu \mathrm{g} / \mathrm{kgKG}$ verwendet. Bei 1- bis 5-jährigen Kindern konnte die Analgesiedauer des KB mit 0,2\%igem Ropivacain durch die Zugabe von $2 \mu \mathrm{g} / \mathrm{kgKG}$ Clonidin von 291 min auf 492 min verlängert werden [39]. Der sedierende Nebeneffekt von Clonidin reduziert die Inzidenz eines Aufwachdelirs, ist aber auch ein Grund, weshalb in der ambulanten Chirurgie gelegentlich auf Clonidin verzichtet wird. Bei kleinen Säuglingen, insbesondere bei ehemals Frühgeborenen, können nach kaudaler Clonidinapplikation Apnoen auftreten. In der Institution der Autoren wird deshalb bei Säuglingen $<3$ Monaten und bei ehemals Frühgeborenen mit $<60$ Gestationswochen auf den Clonidinzusatz bei Anlage eines KB verzichtet. Andere Einrichtungen verwenden Clonidin bei Patienten in einem Alter $>6$ Monate oder mit einem Körpergewicht $>10 \mathrm{~kg}$ [26]. Anhand der Literatur ist diesbezüglich derzeit keine evidenzbasierte Empfehlung möglich.

Opiate. Die Analgesie des KB wird einzig durch Morphin in klinisch relevanter Weise verlängert. Nebenwirkungen wie

- Nausea,

- Pruritus,

- Harnretention und

- Atemdepression
Auf keinen Fall darf Propofol als Ersatz des Lipidverfahrens beim lokalanästhetikainduzierten Herz-Kreislauf-Kollaps verwendet werden

Durch den Einsatz von Adjuvanzien können die Gesamtdosis des Lokalanästhetikums und dosisabhängige Nebenwirkungen reduziert werden

Bei kleinen Säuglingen können nach kaudaler Clonidinapplikation Apnoen auftreten 
Opiatzusätze zum Kaudalblock haben sich in der klinischen Routine nicht durchgesetzt

Ketamin wird auch heute noch von Experten als Adjuvans empfohlen sind jedoch nicht selten. Entsprechend muss bei ambulanten Eingriffen von Morphin als Zusatz zum KB abgeraten werden [40]. Opiatzusätze zum KB haben sich in der klinischen Routine nicht durchgesetzt [8] und bleiben Einzelfällen vorbehalten.

Ketamin. Die epidurale Gabe von 0,5-1 mg/kgKG S-Ketamin führt zu einer relevanten Wirkungsverlängerung des LA um mehrere Stunden: Der Zusatz von 0,5 mg/kgKG S-Ketamin zu 0,2\%igem Ropivacain bewirkte eine Verlängerung der Analgesiedauer von $291 \mathrm{~min}$ auf $701 \mathrm{~min}$ [39]. Aufgrund dieses deutlichen Effekts wurde Ketamin oft als Ergänzung zum KB verwendet [8]; auch heute noch wird es von Experten empfohlen [40]. Andererseits liegen Berichte über die Neurotoxizität von Ketamin vor [41], sodass viele Anästhesisten aus Sicherheitsgründen wieder davon abraten [5, 13, 42].

Midazolam und Neostigmin. Aufgrund der beschränkten Datenlage kann keine Empfehlung für den klinischen Einsatz gegeben werden [5, 13].

\section{Fazit für die Praxis}

- Der KB ist nach wie vor die häufigst durchgeführte Regionalanästhesietechnik zur perioperativen Analgesie in der Kinderanästhesie und zudem ein sehr sicheres Verfahren. Meist wird er beim schlafenden Kind angelegt.

- Für optimalen Erfolg und Sicherheit müssen bei der Durchführung technische Details beachtet werden. Der Prävention und Früherkennung einer systemischen LA-Intoxikation gebührt dabei besondere Aufmerksamkeit.

- Die langsame Injektion des LA unter intermittierender Aspirations-/Rückflussprobe ist obligatorisch, um eine intravasale Injektion primär zu verhindern bzw. hohe Plasmaspiegel bei akzidenteller intravasaler Injektion zu vermeiden.

- Neben dem LA sollte Adrenalin als Marker einer akzidentellen intravaskulären LA-Injektion verwendet werden.

- Eine kontinuierliche EKG-Überwachung und 1-minütliche Blutdruckmessung sind bei der LA-Injektion obligatorisch.

- Im Fall einer akuten LA-induzierten Herz-Kreislauf-Depression bzw. eines Herz-Kreislauf-Kollapses ist Adrenalin weiterhin die unverzichtbare Erstlinienmedikation.

- Die Lipidtherapie ist kein Ersatz für Adrenalin. Sie hilft als ergänzende Maßnahme, den Kreislauf zu stabilisieren.

\section{Korrespondenzadresse}

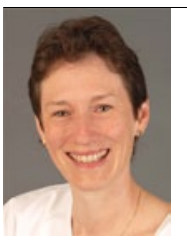

Dr. J. Mauch

Anästhesieabteilung, Universitäts-Kinderkliniken Zürich

Steinwiesstr. 75, 8032 Zürich

jacqueline.mauch@kispi.uzh.ch

Interessenkonflikt. Die korrespondierende Autorin gibt für sich und ihren Koautor an, dass kein Interessenkonflikt besteht. 


\section{Literatur}

1. Ecoffey C, Lacroix F, Giaufré E et al (2010) Epidemiology and morbidity of regional anaesthesia in children: a follow-up one-year prospective survey of the FrenchLanguage Society of Paediatric Anaesthesiologists (ADARPEF). Pae diatr Anaesth 20:1061-1069

2. Gunter J (1991) Caudal anaesthesia in children: a survey. Anesthesiology 75:A936

3. Giaufré $E$, Dalens $B$, Gombert A (1996) Epidemiology and morbidity of regional anaesthesia in children: a one-year prospective survey of the French-Language Society of Paediatric Anaesthesiologists. Anesth Analg 83:904-912

4. Veyckemans F, Van Obbergh $\mathrm{L}$ Gouverneur JM (1992) Lessons from 1100 pediatric caudal blocks in a teaching hospital. Reg Anesth 17:119-1125

5. Mader T, Hornung M, Boos K et al (2007) Handlungsempfehlungen zur Regionalanästhesie bei Kindern. Anaesth Intensivmed 48:7985

6. Veyckemans F, Scholtes JL (2007) Caudal block and ventricular shunt devices: beware of the consequences of increasing epidural pressure! Paediatr Anaesth 17:707-709

7. Mauch JY, Spielmann N, Hartnack S, Weiss M (2012) Electrocardiographic and haemodynamic alterations caused by three different test solutions of local anaesthetics to detect accidental intravascular injection in children. $\mathrm{Br} \mathrm{J}$ Anaesth 108:283-289

8. Menzies R, Congreve $K$, Herodes V et al (2009) A survey of pediatric caudal extradural anesthesia practice. Paediatr Anaesth 19:829-836

9. Baris S, Guldogus F, Baris YS et al (2004) Is tissue coring a real problem after caudal injection in children. Paediatr Anaesth 14:755-758

10. Wittum S, Hofer CK, Rölli U et al (2003) Sacral osteomyelitis after single-shot epidural anesthesia via the caudal approach in a child. Anesthesiology 99:503-505
11. Birnbach DJ, Meadows W, Stein DJ et al (2003) Comparison of povidone iodine and DuraPrep, an iodophor-in-isopropyl alcohol solution, for skin disinfection prior to epidural catheter insertion in parturients. Anesthesiology 98:164-169

12. Kinirons B, Mimoz O, Lafendi L et al (2001) Chlorhexidine versus povidone iodine in preventing colonization of continuous epidural catheters in children: a randomized, controlled trial. Anesthesiology 94:239-244

13. Jöhr M, Berger TM (2012) Caudal blocks. Paediatr Anaesth 22:44-50

14. Gerber AC, Weiss M (2002) Das ehemalige Frühgeborene mit Leistenhernien. Welches Anästhesieverfahren? Anaesthesist 51:448-456

15. Hoelzle M, Weiss M, Dillier C, Gerber $A$ (2010) Comparison of awake spinal with awake caudal anesthesia in preterm and ex-preterm infants for herniotomy. Paediatr Anaesth 20:620-624

16. Bösenberg A (2011) Regional anesthesia: central neuraxial blocks. In: Bissonnette B (Hrsg) Pediatric anesthesia. Basic principles - state of the art - future. People's Medical Publishing House, Shelton, S 815816

17. Lönnqvist PA (2010) Is ultrasound guidance mandatory when performing paediatric regional anaesthesia? Curr Opin Anaesthesiol 23:337-341

18. Fisher QA, Shaffner DH, Yaster M (1997) Detection of intravascular injection of regional anaesthetics in children. Can J Anaesth 44:592598

19. Mauch J, Kutter AP, Martin Jurado 0 et al (2011) Influence of bupivacaine injection dose rate on cardiovascular depression, subsequent hemodynamic course, and related bupivacaine plasma levels in piglets. J Anesth 25:710-715

20. Eastwood D, Williams C, Buchan I (1998) Caudal epidurals: the whoosh test. Anaesthesia 53:305 307

21. Dearlove O, Walker RWM, Bigeon JY (1998) The whoosh test and caudal anaesthesia. Anaesthesia 53:829
22. Saberski LR, Kondamuri S, Osinubi OY (1997) Identification of the epidural space: is loss of resistance to air a safe technique? A review of the complications related to the use of air. Reg Anesth 22:3-15

23. Dobereiner EFA, Cox RG, Ewen A, Lardner DR (2010) Evidence-based clinical update: Which local anesthetic drug for pediatric caudal block provides optimal efficacy with the fewest side effects? Can J Anaesth 57:1102-1110

24. Morrison SG, Dominguez JJ, Frascarolo P, Reiz S (2000) A comparison of the electrocardiographic cardiotoxic effects of racemic bupivacaine, levobupivacaine, and ropivacaine in anesthetized swine. Anesth Analg 90:1308-1314

25. Santos AC, DeArmas PI (2001) Systemic toxicity of levobupivacaine, bupivacaine, and ropivacaine during continuous intravenous infusion to nonpregnant and pregnant ewes. Anesthesiology 95:12561264

26. Philippi-Hoehne C (2012) Perioperative Schmerztherapie bei Kindern. Anaesth Intensivmed 53:3344

27. Hübler $M$, Gäbler $R$, Ehm B et al (2010) Successful resuscitation following ropivacaine-induced systemic toxicity in a neonate. Anaesthesia 65:1137-1140

28. Lin EP, Aronson LA (2010) Successful resuscitation of bupivacaine-induced cardiotoxicity in a neonate. Paediatr Anaesth 20:955-957

29. Mauch J, Kutter AP, Madjdpour C et al (2010) Electrocardiographic changes during continuous intravenous application of bupivacaine in neonatal pigs. $\mathrm{Br} \mathrm{J}$ Anaesth 105:437-441

30. Neal JM, Bernards CM, Butterworth JF et al (2010) ASRA practice advisory on local anesthetic systemic toxicity. Reg Anesth Pain Med 35:152-161

31. Tobias JD (2001) Caudal epidural block: a review of test dosing and recognition of systemic injection in children. Anesth Analg 93:11561161
32. Polaner DM, Zuk J, Luong K, Pan Z (2010) Positive intravascular test dose criteria in children during total intravenous anesthesia with propofol and remifantanil are different than during inhaled anesthesia. Paediatr Anaesth 110:41-45

33. Zausig YA, Zink W, Graf BM (2012) What limits the effect of lipid emulsion therapy? Eur J Anaesthesiol 29:159-160

34. Spence AG (2007) Lipid reversal of central nervous system symptoms of bupivacaine toxicity. Anesthesiology 107:516-517

35. Whiteside J (2008) Reversal of local anaesthetic induced CNS toxicity with lipid emulsion. Anaesthesia 63:203-204

36. Mauch J, Martin Jurado O, Spielmann $\mathrm{N}$ et al (2011) Comparison of epinephrine vs lipid rescue to treat severe local anesthetic toxicity - an experimental study in piglets. Paediatr Anaesth 21:1103-1108

37. Mauch J, Martin Jurado O, Spielmann N et al (2012) Resuscitation strategies from bupivacaine-induced cardiac arrest. Paediatr Anaesth 22:124-129

38. Bouaziz H, Okubo N, Malinovsky JM et al (1999) The age-related effects of epidural lidocaine, with and without epinephrine, on spinal cord blood flow in anesthetized rabbits. Anesth Analg 88:1302-1307

39. De Negri $P$, Ivani $G$, Visconti $C$, De Vivo $P$ (2001) How to prolong postoperative analgesia after caudal anaesthesia with ropivacaine in children: S-ketamine versus clonidine. Paediatr Anaesth 11:679-683

40. Lönnqvist PA (2011) Blocks for pain management in children undergoing ambulatory surgery. Curr Opin Anaesthesiol 24:627-632

41. Braun S, Gaza N, Werdehausen $R$ et al (2010) Ketamine induces apoptosis via the mitochondrial pathway in human lymphocytes and neuronal cells. $\mathrm{Br} J$ Anaesth 105:347-354

42. Becke K, Höhne C, Jöhr M, Reich A (2007) Stellungnahme des wissenschaftlichen Arbeitskreises Kinderanästhesie der DGAI zum kaudalen Einsatz von S(+)-Ketamin. Anaesth Intensivmed 48:298-299 
Bitte beachten Sie:

- Teilnahme nur online unter: springermedizin.de/eAkademie

- Die Frage-Antwort-Kombinationen werden online individuell zusammengestellt.

- Es ist immer nur eine Antwort möglich.

Welche Aussage zum Kaudalblock (KB) in der Kinderanästhesie ist richtig?

Der KB wird nur in Ausnahmefällen mit der Allgemeinanästhesie kombiniert.

$\square$ Der KB sollte bei Säuglingen nicht angewendet werden.

$\square$ Der KB ist eine sichere und rasch erlernbare Technik.

$\square$ Der KB erfordert keine Aufklärung/Einwilligung der Eltern.

$\square$ Der KB bewirkt eine auf den Gesäßbereich begrenzte Analgesie.

? Was ist eine obligatorische Voraussetzung für die Anlage eines KB?

$\square$ Ein dokumentierter Gerinnungsstatus bei blander Anamnese.

$\square$ Ein Ausschluss eines viralen Infekts der oberen Luftwege.

$\square$ Eine Nadel mit Stilett.

$\square$ Ein Ultraschallgerät.

$\square$ Eine Herz-Kreislauf-Überwachung (EKG, nichtinvasive Blutdruckmessung).

? Welche Aussage zur Anlage des KB ist falsch?

$\square$ Das Tragen steriler Handschuhe ist Standard.

$\square$ Die Desinfektion mit einem alkoholhaltigen Mittel wird empfohlen.

$\square$ Die Einwirkzeit des Desinfektionsmittels muss abgewartet werden.

$\square$ Die Punktion im kaudalen Anteil des Hiatus sacralis ist am erfolgversprechendsten.

$\square$ Die Linksseitenlage des Patienten empfiehlt sich für den rechtshändig punktierenden Anästhesisten.

\section{Welche Aussage zur Anlage des KB ist} richtig?

$\square$ Die altersabhängige Lage des Duralsackendes ist zu berücksichtigen.

$\square$ Nach Perforation des Lig. sacrococcygeum wird die Nadel $1-2 \mathrm{~cm}$ vorgeschoben.

$\square$ Das Os sacrum kann bei Säuglingen mit einer Nadel nicht penetriert werden.

$\square$ Eine akzidentelle intraossäre Medikamentenapplikation hat keine relevanten Fol-

\section{CME-Fragebogen \\ kostenfreie Teilnahme am e.CME für Zeitschriftenabonnenten}

gen auf Lokalanästhetika(LA)-Plasmaspiegel.

Als LA (in Kombination mit einer Allgemeinanästhesie) wird meist Bupivacain $0,5 \%$ verwendet.

? Welchen Vorteil im Vergleich zu Bupivacain hat Ropivacain bei Verwendung beim KB?

$\square$ Bessere intraoperative Analgesie.

$\square$ Weniger intraoperative hämodynamische Veränderungen.

$\square$ Längere postoperative Analgesie.

Weniger postoperative Übelkeit und Erbrechen.

Geringere motorische Blockade.

Welches Adjuvans weist den besten Effekt hinsichtlich der Wirkungsverlängerung und -intensivierung bei geringsten unerwünschten Nebenwirkungen beim KB auf?

$\square$ Adrenalin

$\square$ Clonidin.

$\square$ Morphin.

$\square$ Ketamin.

Midazolam.

? Durch welche Maßnahme kann eine systemische LA-Intoxikation zuverlässig verhindert werden?

$\square$ Einhaltung der empfohlenen Maximaldosierungen.

$\square$ Verwendung von Ropivacain anstelle von Bupivacain.

$\square$ LA-Applikation unter Ultraschallkontrolle.

$\square$ Aspirationstest zum Ausschluss einer intravasalen Lage der Kanüle.

Keine einzelne Maßnahme verhindert eine LA-Intoxikation mit Sicherheit.

? Welche Aussage zur akzidentellen intravasalen LA-Injektion ist richtig?

$\square$ Sie kann durch den Aspirationstest vor Injektion des LA ausgeschlossen werden.

Sie kann durch einen Adrenalinzusatz frühzeitig erkannt werden.
Sie hat abgesehen von einem möglicherweise reduzierten Effekt der Regionalanästhesie keine klinische Bedeutung.

Die Spritzgeschwindigkeit hat keinen Einfluss auf die Plasmaspiegel des LA.

Sie ist in der Kinderanästhesie kein Thema.

Welche Aussage zur intravasal applizierten adrenalinhaltigen LA-Testdosis ist richtig?

Die kardiovaskulären Effekte sind in allen Altersgruppen gleich.

$\square$ Die kardiovaskulären Effekte treten erst ab einer Adrenalindosis von $5 \mu \mathrm{g} / \mathrm{kgKG}$ auf.

Typisch ist eine Verbreiterung des QRSKomplexes.

$\square$ Eine Erhöhung von T-Wellen ist bei Vorschulkindern ein zuverlässiges positives Zeichen.

$\square$ Eine Reduktion der Herzfrequenz tritt nicht auf.

? Welche Aussage zur kardiopulmonalen Reanimation infolge einer LA-Intoxikation ist richtig?

Adrenalin gilt als Erstlinientherapie.

$\square$ Eine"lipid rescue" hat absolute Priorität.

Propofol stellt einen Ersatz für eine Lipidemulsion dar, falls diese nicht verfügbar ist.

Der Wirkmechanismus der Lipidemulsion ist abschließend geklärt und bekannt.

$\square$ Die aktuellen Reanimationsleitlinien sind von untergeordneter Bedeutung.

Diese zertifizierte Fortbildung ist 12 Monate auf springermedizin.de/ eAkademie verfügbar. Dort erfahren Sie auch den genauen Teilnahmeschluss. Nach Ablauf des Zertifizierungszeitraums können Sie diese Fortbildung und den Fragebogen weitere 24 Monate nutzen. 